\title{
La Gestión de Recursos Humanos. Últimas Tendencias y Modelo Diferencial en España ${ }^{(*)}$
}

\author{
Fernando Matias Reche \\ Victor.Jesuis García Morales \\ Universidad de Granada
}

RESUMEN

En el presente trabajo pretendemos poner de manifiesto la evoluciön seguida por la Gestion de Recursos Humanos en los íltimos años, las ültimas tendencias en este campo y si en España se sigue un modelo de gestión de personal diferenciado del seguido a nitel internacional. Para cumplir con el anterior propósito bemos realizado un estudio sobre la frecuencia de aparición de determinados tópicos tradicionales y recientes de la Gestión de Recursos Humanos en las principales revistas de esta disciplina a nivel internacional y nacional.

Palabras clave: Úttimas tendencias, Modelo diferencial. Inuestigación.

\section{ABSTRACT}

This work seeks to show the evolution of human resources management in the last years, the last tendencies in this field and if Spain bas a different model of the folloued to international level. To fulfill the previous purpose we bave carried out a study about the frequency of appearance of certain traditional and recent topics of the human resources management in the main magazines from this discipline to international and national level.

Key words: Last tendencies, Diferential model, Research.

(*) Este trabajo ha sido financiado por el Proyecto de Investigación "Cambios mediambientales en las empresas: Gestión de los recursos humanos y organizativos para la generación de lcapacidades competitivas sostenibles". referencia del Ministerio de Ciencia y Tecnología: SEC2000-1479-C03-01. 


\section{INTRODUCCION}

En el presente trabajo pretendemos poner de manifiesto la evolución seguida por la Gestión de Recursos Humanos en los últimos años, las últimas tendencias en este campo y si en España se sigue un modelo de gestión de personal diferenciado del seguido a nivel internacional. Para cumplir con el anterior propósito hemos realizado un estudio sobre la frecuencia de aparición de determinados tópicos tradicionales y recientes de la Gestión de Recursos Humanos en las principales revistas de esta disciplina a nivel internacional y nacional.

La función de Gestión de los Recursos Humanos se ha desarrollado durante los últimos cuarenta años, y cada década ha incluido una nueva serie de herramientas de gestión: las relaciones laborales y contratación (años cuarenta); la formación (años cincuenta): las cuestiones reguladoras, la compensación, los beneficios sociales, la evaluación (años sesenta y setenta); la preocupación por la salud, la contención de costes, el diseño de la organización, el trabajo en equipo y la comunicación (años ochenta); las fusiones. las adquisiciones, la reducción de plantilla, la diversidad (años noventa).

Habida cuenta de la evolución en las herramientas, puede ser interesante explorar dicha evolución, y las últimas tendencias de investigación dentro de este campo, tanto a nivel internacional como nacional; a la vez, algunas referencias como Miranda (1995), donde se consideran diferentes niveles de desarrollo y uso de las empresas de trabajo temporal en Estados Unidos, en concreto, comenta que en este país ya están en la tercera generación, cuando en España aún estamos en la primera, o Quintanilla (1991: pp. 22-24), quien aludiendo a las diferencias culturales, históricas, etc. de España intenta justificar los distintos talantes de dirección, de medida de la eficacia o de la manera de hacer del trabajador español respecto al de otros países (Estados Unidos, Japón, Dinamarca, etc.), hacen interesante comprobar si en España seguimos o por el contrario, nos diferenciamos de las corrientes seguidas a nivel internacional en el campo de la Gestión de los Recursos Humanos.

\section{Metodología}

Para los anteriores propósitos hemos consultado la principal revista de Gestión de Recursos Humanos a nivel internacional (Human Resource Management (HRM)) y la revista más importante en España, que trata exclusivamente la problemática de la gestión de personal durante un período relativamente amplio (Capital Humano $(\mathrm{CH}))^{2}$. Para rastrear la revista Human Resource Management hemos utilizado la

1. Según Román (2000), esta revista es la que cuenta con un mayor número de artículos que responden a los descriptores "HRM" o "Human Resource Management" de entre las principales revistas internacionales del área de management. 
base de datos Social Science Citation Index (SSCI), y para la revista Capital Humano, hemos utilizado la base de datos ISOC del Consejo Superior de Investigaciones Científicas (CSIC). Para ambas revistas el período de estudio es de 1991 a 1999 , ambos inclusive.

Del análisis de la anterior búsqueda obtuvimos una muestra de 1.193 artículos, de los cuales 249 (20,9\%) son de Human Resource Management y 944 (79,1\%) son de Capital Humano. La distribución de artículos por años en ambas revistas muestran que el año con más artículos consultados es 1994 (168) lo que representa un $14,1 \%$ del total y el año con un menor número es 1998 (9,1\%). Por revistas, es también 1994 cuando aparecen más artículos en la revista Capital Humano (138), siendo su menor número en 1998 (88). Para la revista Human Resource Management, 1996 es el año con más artículos (44) y 1991 y 1998 los años con menos (ver cuadro 1).

Cuadro 1: DISTRIBUCIÓN DE ARTÍCULOS POR AÑO Y REVISTAS

\begin{tabular}{|l|l|l|l|l|l|l|l|l|l|l|l|}
\hline & $\mathbf{1 9 9 1}$ & $\mathbf{9 9 2}$ & $\mathbf{1 9 9 3}$ & $\mathbf{1 9 9 4}$ & $\mathbf{1 9 9 5}$ & $\mathbf{1 9 9 6}$ & $\mathbf{1 9 9 7}$ & $\mathbf{1 9 9 8}$ & $\mathbf{1 9 9 9}$ & Total & $\%$ \\
\hline HRM & 21 & 22 & 25 & 30 & 27 & 24 & 44 & 21 & 35 & 249 & 20,9 \\
\hline CH & 94 & 93 & 90 & 138 & 130 & 107 & 111 & 88 & 93 & 944 & 79,1 \\
\hline Total & 115 & 115 & 115 & 168 & 157 & 131 & 155 & 109 & 128 & 1.193 & 100 \\
\hline$\%$ & 9,6 & 9,6 & 9,6 & 14,1 & 13,2 & 11,0 & 13,0 & 9,1 & 10,7 & 100 & \\
\hline
\end{tabular}

El procedimiento para clasificar los artículos consultados es similar al empleado por Roman (2000). En este caso, las tres categorías de tópicos consideradas son: Tópicos Tradicionales, Tópicos Recientes, Otros Temas. En el primer grupo se incluyeron aquellos artículos que trataban sobre las funciones que tradicionalmente han sido desempeñadas por la unidad experta en la Gestión de Recursos Humanos. El segundo grupo incorpora los artículos que mejor vienen a caracterizar la nueva Gestión de Recursos Humanos, es decir, el resto de funciones, retos o cambios que hoy día está afrontando la función de personal para apoyar la adaptación a un entorno caracteriza-

2. Según Dolan, Schuler y Valle (1999: p. 416), esta revista está entre las principales revistas y publicaciones de recursos humanos en España. 
do por la competitividad. la globalización, el cambio continuo de los mercados y las nuevas tecnologías. En el último grupo incluimos aquellos trabajos no considerados en las dos categorias anteriores, como evolución de la función de Gestión de Recursos Humanos. Teoria de la Organización y Gestión de los Recursos Humanos, técnicas de gestión empresarial, acción social, empleo, empresas familiares, etc. El método para clasificar los artículos en cualquiera de los tópicos considerados consistía en analizar el trabajo a partir del título. descriptores o palabras clave y el abstrac, incluso en algunos casos revisamos el artículo completo. Una vez analizados estos elementos, ya contábamos con la información necesaria para poder proceder a la clasificación del trabajo.

El proceso de clasificación se realizó dos veces de forma autónoma, es decir sin tener en cuenta en la segunda vez como se había clasificado la primera. Una tercera vez se realizó la clasificación, en este caso, teniendo en cuenta las discrepancias observadas en las rondas anteriores y asignando estos artículos a la categoría sobre la que más se insistía o era el núcleo del trabajo.

En el cuadro 2 aparece una descripción de los tópicos tradicionales clasificados en dos subgrupos: aquellos relacionados con la función de dirección de las personas (liderazgo. comunicación, equipos de trabajo, etc.) y los que tratan sobre los procesos o prácticas de Gestión de Recursos Humanos (planificación, selección, formación, etc.). Los contenidos tenidos en cuenta a la hora de clasificar los artículos en los tópicos tradicionales a los que más frecuentemente se ha hecho mención en el estudio realizado son los siguientes:

- Liderazgo. En este tópico hemos incluido aquellos artículos que hacían mención a las caracteristicas y habilidades que debe poseer el líder, estilos de liderazgo (liderazgo participativo y otros), etc.

- Comunicación. Hemos incluido los diversos aspectos relacionados con este tópico, en muchos casos unidos a la calidad total, pero de la que hemos intentado deslindarla. Además, es importante hacer mención a un número monográfico de la Human Resource Management (1998, Vol. 37, N. 3-4) dedicado a este tema.

- Selección. En este tópico hemos incluido aquellos aspectos tenidos en cuenta para proceder a la selección de empleados y directivos, entre ellos los relacionados con la inteligencia emocional. La revista Capital Humano dedica parte de un suplemento a tratar este proceso de Gestión de Recursos Humanos (1995, N. 83, pp. 36-58).

- Formación. En este caso hemos incluido aquellos artículos relacionados con el aprendizaje y formación, tanto de empleados y como de directivos, siendo un tema ampliamente tratado en las revistas consultadas.

- Retribución y prestaciones sociales. En este tópico hemos incluido aquellos artículos relacionados con la retribución, tanto directa como indirecta de empleados y directivos, incluyendo aspectos como los problemas de agencia, los planes de pensiones colectivos, etc. 


\section{Cuadro 2. TÓPICOS TRADICIONALES EN LA GESTIÓN DE LOS RECURSOS HUMANOS}

\section{Dirección}

Liderazgo

Comunicación

Grupos-Equipos de Trabajo

Motivación

Satisfacción

Estrés laboral

Negociación laboral

Regulación laboral

Rotación de personal
Selección

\section{Procesos de Gestión Recursos Humanos}

Diseño y análisis de puestos

Planificación de personal

Contratación

Formación

Gestión de carreras

Valoración del rendimiento

Retribución y prestaciones sociales

Prevención de riesgos laborales

Auditoría de la función recursos humanos

Fuente: Adaptado de Román (2000: p. 248)

En cuanto a los tópicos recientes, son cada vez más los retos ante los que se enfrentan los directores de recursos humanos en las organizaciones y como señala Ulrich (1997), habrá nuevas herramientas para los recursos humanos que se concentrarán en áreas como los recursos humanos globales (aprender a dirigir las cuestiones de los recursos humanos en una competencia global), el cambio de cultura (definir las herramientas para construir y cambiar una cultura de la empresa), la tecnología (adaptar 
los recursos humanos a las siempre cambiantes autopistas de la información), el líder del futuro (definir las competencias del próximo líder, no del anterior) y la transmisión de conocimientos (entender cómo generar y generalizar los conocimientos). Éstos y otros retos constituyen tendencias o líneas de investigación que se están desarrollando en la actualidad en el área de los recursos humanos y que han sido clasificadas para nuestro análisis como se refleja en el cuadro 3.

Los contenidos tenidos en cuenta para clasificar un artículo en algunos de los tópicos recientes más tratados son los siguientes:

- Gestión estratégica de recursos humanos. En este tópico se han incluido los trabajos sobre la conexión entre los procesos de Gestión de Recursos Humanos y la estrategia de la empresa en su conjunto, así como aquellos que hacían mención a la consideración de los recursos humanos como elemento importante para conseguir una ventaja competitiva sostenible. Sobre este tópico, la Human Resource Management publica un número monográfico sobre cinco casos de empresas líderes (1999, Vol. 38, N. 4).

- Gestión internacional de recursos humanos. Los artículos clasificados aquí hacen referencia a la problemática planteada en las empresas a la hora de gestionar su personal en términos globales, haciendo mención a los expatriados y a su situación cuando van o regresan de ocupar puestos en filiales o sucursales en el extranjero. También se incluyen es este tópico los artículos que comparaban la gestión de recursos humanos en distintos países o se referían a las características de su gestión en países del lejano oriente (Japón, Corea, Singapur, etc.).

- Las nuevas funciones de la Gestión de Recursos Humanos y competencias de sus líderes. A la hora de abordar los contenidos a incluir en este tópico nos decantamos por incluir todos aquellos relacionados con las nuevas competencias que debe de poseer el director de recursos humanos, así como la nuevas funciones de las que se tendrá que hacer responsable. Se trata de desempeñar, principalmente, el rol de "catalizadores del cambio", permitiendo que la organización se adapte al entorno gracias, entre otros elementos, a las personas que en ella operan, a su cualificación, a la flexibilidad de horarios, en las modalidades de trabajo, etc ${ }^{3}$. Aunque distintas de las clásicas o tradicionales, estas nuevas funciones son complementarias a ellas. La Human Resource Management tiene un número especial dedicado al futuro de la Gestión de Recursos Humanos (1997, Vol. 36, N. 1).

- Gestión por competencias. Esta nueva forma de gestionar los recursos humanos está teniendo cada vez un mayor interés por parte de los estudiosos sobre esta disciplina. Así, las competencias o algunas de ellas consideradas clave se convierten en el

3. En Matias (2000) aparece un elenco de los distintos tipos de flexibilidad laboral y modalidades de empleo según un modelo de empresa flexible. 
núcleo sobre el que giran los procesos de Gestión de Recursos Humanos. También sobre este tema, la revista Human Resource Management tiene publicado un número monográfico (1996, Vol. 35, N. 3).

- Cultura organizativa y Gestión de Recursos Humanos. Dentro de este tópico se han incluido aquellos artículos que hacian referencia a la gestión por valores, cambio cultural y el papel que la Gestión de Recursos Humanos juega en esos aspectos.

- Calidad y Gestión de Recursos Humanos. Pocos temas han recibido tanta atención en los últimos años en el mundo empresarial como el movimiento de la Gestión de la Calidad Total. En su relación con la gestión de personal, se han incluido en este tópico aquellos trabajos relacionados con las características, competencias y necesidades de los empleados para implantar la Gestión de la Calidad Total en las empresas.

- Cambio organizativo y los recursos humanos. Al igual que con la Gestión de la Calidad, en este caso, hemos considerado como pertenecientes a este tópico los artículos relacionados con el papel que juegan los recursos humanos en el cambio organizativo, incluyendo temas relacionados con la organización que aprende o el clesarrollo organizacional.

- Flexibilidad laboral. En la última década, los gobiernos de los países de nuestro entorno y por supuesto el de Espana, junto con los agentes sociales se han hecho eco de la necesidad de una mayor flexibilidad en las relaciones laborales. A este respecto han articulado medidas y acuerdos para ampliar los distintos tipos de flexibilidad laboral: numérica, funcional, del tiempo de trabajo, etc. Por lo tanto, en este tópico hemos incluido todos los trabajos relacionados con los anteriores tipos de flexibilidad.

- Nuevo contrato psicológico. En este tópico incluimos los artículos relacionados con la necesidad de un nuevo tipo de contrato psicológico entre empleador y empleado tras la transformación del sistema tradicional de empleo estable en otro donde ya no se garantiza dicha estabilidad, pero sí se requiere el compromiso del trabajador con la empresa.

- Dounsizing. En este tópico hemos incluido aquellos artículos relacionados con la disminución del tamaño de la plantilla de la empresa en busca de una mayor flexibilidad. Igualmente, se han incluido en este tópico los artículos que trataban sobre el outplacement o recolocación de empleados. Sobre este tema hay un suplemento en la revista Capital Humano dedicado, en parte, él (1995, N. 83, pp. 6-34), y sobre Downsizing, la Human Resource Management, igualmente, editó un número monográfico (1994, Vol. 33, N. 2).

- Tecnologías de la información. En este tópico hemos incluido los trabajos relacionados con este importante fenómeno de nuestro tiempo, que también afecta de forma muy importante a la Gestión de Recursos Humanos, convirtiéndose en una de sus herramientas más importantes. 


\section{Cuadro 3. \\ TÓPICOS RECIENTES EN LA GESTIÓN DE LOS RECURSOS HUMANOS}

Gestión estratégica de recursos humanos:

- Los recursos humanos como fuente de ventaja competitiva.

- Los procesos y la estrategia de la empresa.

Gestión internacional de los recursos humanos:

- Gestión global de recursos humanos

- Países del lejano oriente

- Expatriados

Cambios demográficos en la mano de obra:

- Personas de edad avanzada- Diversidad cultural

Flexibilidad laboral

- Flexibilidad de horarios

- Contratos temporales

- Teletrabajo

Nuevas funciones de la Gestion de Recursos Humanos y competencias de sus líderes

Gestión del conocimiento

Gestión por competencias

Cultura organizativa y Gestión de los Recursos Humanos

Calidad y Gestión de los Recursos Humanos

Mujeres directivas

Fusiones, adquisiciones y los recursos humanos

Cambio organizativo y los recursos humanos

Gestión de los Recursos Humanos y resultados de la empresa

Nuevo contrato Psicológico 


\section{Dounsizing}

Tecnologías de la información

Trabajo y familia.

Fuente: Adaptado de Román (2000: p. 249)

En cuanto a las técnicas utilizadas en este estudio, se han empleado las clásicas estadísticas descriptivas para recoger de forma sintética los resultados sobre la evolución de los distintos tópicos de la Gestión de Recursos Humanos. Además, el test de la $\mathrm{x}^{2}$ de Pearson es empleado para contrastar las posibles diferencias en el interés por los mencionados tópicos a nivel internacional y nacional.

\section{RESUltados}

Los resultados muestran como del conjunto de tópicos, al que más se hace alusión por los investigadores en Gestión de Recursos Humanos es al de "formación", seguido por la "calidad y la Gestión de Recursos Humanos" y el "liderazgo". Entre los tópicos menos tratados está la "rotación de personal", "la planificación de personal" y "la contratación".

Si consideramos la distribución del conjunto de tópicos según las revistas consultadas (Cuadro 4), los resultados muestran que las "nuevas funciones de la Gestión de Recursos Humanos" es al que más se hace referencia en Human Resource Management, seguido por la "gestión estratégica de recursos humanos" y por la "formación", siendo los años en los que se hace mayor insistencia en cada uno de ellos: 1997 para el primero (publicado un número monográfico dedicado al futuro de la Gestión de Recursos Humanos), 1999 para el segundo (editado un número monográfico dedicado a este tema) y 1999 para el tercero. En la evolución de estos tópicos, la tendencia dibujada por el primer tópico más tratado muestra un pico muy acusado para 1997 , donde se concentran la mayoría de los artículos. Con respecto a la "gestión estratégica de recursos humanos", en su evolución se describe una tendencia creciente desde 1991, mostrando un repunte importante en el último año considerado. La "formación" describe una tendencia muy similar a la seguida por el tópico anterior.

En la revista Capital Humano el tópico más tratado es la "formación", seguido por la "calidad y la Gestión de los Recursos Humanos" y por el "liderazgo". Igualmente, los años en los que más se insiste en estos tópicos son 1994 para el primero, 1995 para el segundo y 1997 para el tercero. El análisis de la evolución de estos tópicos muestra que el primero es tratado de forma relativamente constante. El segundo, tras el máximo de 1995 describe una tendencia descendente, y el tercero dibuja una evolución constante salvo un pico acusado, correspondiente a su máximo. 


\begin{tabular}{|c|c|c|c|c|c|c|}
\hline \multirow[b]{2}{*}{ Tópicos } & \multicolumn{2}{|c|}{ HRM } & \multicolumn{2}{|l|}{$\overline{\mathbf{C H}}$} & \multicolumn{2}{|l|}{ Total } \\
\hline & $\overline{\mathbf{n}}$ & $\%$ & $\mathbf{n}$ & $\%$ & $\mathbf{n}$ & $\%$ \\
\hline Liderazgo & 13 & 5,2 & 62 & 6,7 & 75 & 6,3 \\
\hline Comunicación & 9 & 3,6 & 46 & 5,0 & 55 & 4,6 \\
\hline Grupos-Equipos de Trabajo & 0 & 0 & 18 & 1,9 & 18 & 1.5 \\
\hline Motivación & 2 & 0.8 & 23 & 2,4 & 25 & 2,1 \\
\hline Satisfacción & 1 & 0,4 & 8 & 0,8 & 9 & 0,8 \\
\hline Estrés laboral & 0 & 0 & 6 & 0,6 & 6 & 0,5 \\
\hline Negociación laboral & 4 & 1.5 & 17 & 1,8 & 21 & 1,8 \\
\hline Regulación laboral & 0 & 0 & 26 & 2,8 & 26 & 2,2 \\
\hline Rotación de personal & 0 & 0 & 1 & 0,1 & 1 & 0,1 \\
\hline Diseño y análisis de puestos & 0 & 0 & 8 & 0,8 & 8 & 0,7 \\
\hline Planificación de personal & 1 & $0, \dot{4}$ & 0 & 0 & 1 & 0,1 \\
\hline Selección & 1 & 0,4 & 48 & 5,2 & 49 & 4,1 \\
\hline Contratación & 0 & 0 & 1 & 0,1 & 1 & 0,1 \\
\hline Formación & 18 & 7,2 & 125 & 13,3 & 143 & 12,1 \\
\hline Gestión de carreras & 13 & 5,2 & 24 & 2,5 & 37 & 3,1 \\
\hline Valoración del rendimiento & 14 & 5.6 & 18 & 1,9 & 32 & 2,7 \\
\hline Retribución y prestaciones sociales & 4 & 1,6 & 59 & 6,4 & 63 & 5,3 \\
\hline Prevención de riesgos laborales & 4 & 1,6 & 30 & 3,2 & 34 & 2,8 \\
\hline Auditoría de la función recursos humanos & 1 & 0,4 & 29 & 3,1 & 30 & 2,5 \\
\hline Gestión estratégica de recursos humanos & 21 & 8.5 & 17 & 1,8 & 38 & 3,2 \\
\hline Gestión internacional de los recursos humanos & 10 & 4,0 & 22 & 2,3 & 32 & 2,7 \\
\hline Cambios demográficos en la mano de obra & 8 & 3,2 & 8 & 0,2 & 16 & 1,3 \\
\hline Flexibilidad laboral & 2 & 0,8 & 28 & 2,9 & 30 & 2.5 \\
\hline Nuevas funciones de la GRH y comp. sus líderes & 32 & 13 & 23 & 2,4 & 55 & 4,7 \\
\hline Gestión del conocimiento & 2 & 0,8 & 4 & 0,4 & 6 & 0,5 \\
\hline Gestión por competencias & 7 & 2,8 & 19 & 2,0 & 26 & 2,2 \\
\hline Cultura organizativa y GRH & 12 & 4,8 & 36 & 3,8 & 48 & 4,0 \\
\hline Calidad y Gestión de los Recursos Humanos & 6 & 2,4 & 78 & 8,4 & 84 & 7,0 \\
\hline Mujeres directivas & 4 & 1,6 & 5 & 0,5 & 9 & 0,8 \\
\hline Fusiones, adquisiciones y recursos humanos & 0 & 0 & 5 & 0,5 & 5 & 0,4 \\
\hline Cambio organizativo y los recursos humanos & 7 & 2,8 & 32 & 3,5 & 39 & 3,3 \\
\hline GRH y resultados de la empresa & 8 & 3,2 & 5 & 0,5 & 13 & 1,1 \\
\hline Nuevo contrato psicológico & 11 & 4,4 & 2 & 0,2 & 13 & 1,1 \\
\hline Downsizing & 14 & 5,6 & 18 & 1,9 & 32 & 2,7 \\
\hline Tecnologías de la información & 3 & 1,2 & 39 & 4,1 & .42 & 3,6 \\
\hline Trabajo y familia. & 7 & 2,8 & 0 & 0,0 & 7 & 0,6 \\
\hline Otros temas & 10 & 4,0 & 54 & 5,7 & 64 & 5,4 \\
\hline Total & 249 & 100 & 944 & 100 & 1193 & 100 \\
\hline
\end{tabular}


Si tenemos en cuenta al conjunto de tópicos clasificados en tradicionales, recientes y otros, los primeros son los que gozan de un mayor tratamiento, no obstante éste no es homogéneo para las revistas consultadas. Así, la anterior situación se da en Capital Humano, y todo lo contrario en Human Resource Management, donde se hace un mayor hincapié en los tópicos recientes que en los tradicionales (Cuadro 5).

\section{Cuadro 5. DISTRIBUCIÓN DE TÓPICOS TRADICIONAIES, RECIENTES Y OTROS POR REVISTAS}

\begin{tabular}{|l|l|l|l|l|l|l|l|}
\hline Revista & $\begin{array}{l}\text { Tópicos } \\
\text { Tradicional. }\end{array}$ & $\%$ & $\begin{array}{l}\text { Tópicos } \\
\text { Recientes }\end{array}$ & $\%$ & Otros & $\%$ & Total \\
\hline $\begin{array}{l}\text { Human } \\
\text { Manource } \\
\text { Management }\end{array}$ & 85 & 34 & 154 & 62 & 10 & 4 & 249 \\
\hline $\begin{array}{l}\text { Capital } \\
\text { Humano }\end{array}$ & 549 & 58 & 341 & 36 & 54 & 6 & 944 \\
\hline Total & 634 & 54 & 495 & 41 & 64 & 5 & 1193 \\
\hline
\end{tabular}

La evolución cronológica según los tres conjuntos de tópicos y las revistas consideradas (Cuadro 6) muestra como para los tres tipos de tópicos la evolución es diferente de acuerdo a las revistas consideradas. Respecto a los tópicos tradicionales, el año con mayor número de artículos en Human Resource Management fue 1995 y para Capital Humano fue 1994. Las diferencias más importantes entre estas revistas se dan en 1994 y 1991 donde Capilal Humano supera en 11,4 y 9,1 puntos porcentuales, respectivamente, a Human Resource Management. A partir de 1994 se aprecia una tónica constante en ambas revistas, con algunos altibajos.

En cuanto a los tópicos recientes, 1997 y 1995 son los años en los que aparecen más artículos de Human Resource Management y Capital Humano, respectivamente, siendo estos años donde se aprecian las mayores diferencias entre revistas. El año 1995 es en el que Capital Humano supera a Human Resource Management y 1997 en el que sucede lo contrario. Si se observa la evolución por revistas, se puede apreciar como esta última parece mostrar un comportamiento un tanto errático; sin embargo, 'Capital Humano parece describir una tendencia descendente a partir de 1995. 


\begin{tabular}{|c|c|c|c|c|c|c|}
\hline \multicolumn{7}{|c|}{ Cuadro 6. DISTRIBUGIÓN CRONOLÓGICA POR TÓPICOS Y REVISTAS } \\
\hline \multirow[t]{2}{*}{ Año } & \multicolumn{2}{|c|}{ Tópicos tradicional } & \multicolumn{2}{|c|}{ Tópicos recientes } & \multicolumn{2}{|c|}{ Otros temas } \\
\hline & HRM & $\mathrm{CH}$ & HRM & $\mathrm{CH}$ & HRM & $\mathrm{CH}$ \\
\hline 1991 & 2 & 63 & 16 & 29 & 3 & 2 \\
\hline $\begin{array}{l}\text { \% } \\
\text { Columna }\end{array}$ & 2,4 & 11,5 & 10,4 & 8,5 & 30,0 & 3,7 \\
\hline 1992 & 10 & 62 & 11 & 30 & 1 & 1 \\
\hline $\begin{array}{l}\% \\
\text { Columna }\end{array}$ & 11,8 & 11,3 & 7,1 & 8,8 & 10,0 & 1,9 \\
\hline 1993 & 12 & 48 & 13 & 38 & 0 & 4 \\
\hline $\begin{array}{l}\% \\
\text { Columna }\end{array}$ & 14,1 & 8,7 & 8,4 & 11,1 & $\mathbf{0 , 0}$ & 7,4 \\
\hline 1994 & 3 & 82 & 26 & 46 & 1 & 10 \\
\hline $\begin{array}{l}\% \\
\text { Columna }\end{array}$ & 3,5 & 14,9 & 16,9 & 13,5 & 10,0 & 18,4 \\
\hline 1995 & 14 & 58 & 12 & 65 & 1 & 7 \\
\hline $\begin{array}{l}\% \\
\text { Columna }\end{array}$ & 16,4 & 10,6 & 7,8 & 19,1 & 10,0 & 13,0 \\
\hline 1996 & 9 & 63 & 13 & 39 & 2 & 5 \\
\hline $\begin{array}{l}\% \\
\text { Columna }\end{array}$ & 10,6 & 11,5 & 8,4 & 11,4 & 20,0 & 9,3 \\
\hline 1997 & 12 & 73 & 32 & 33 & 0 & 5 \\
\hline $\begin{array}{l}\% \\
\text { Columna }\end{array}$ & 14,1 & 13,3 & 20,9 & 9,7 & $\mathbf{0 , 0}$ & 9,3 \\
\hline 1998 & 10 & 50 & 11 & 35 & 0 & 3 \\
\hline $\begin{array}{l}\% \\
\text { Columna }\end{array}$ & $\mathbf{1 1 , 8}$ & 9,1 & 7,1 & 10,3 & $\mathbf{0 , 0}$ & 5,6 \\
\hline 1999 & 13 & 50 & 20 & 26 & 2 & 17 \\
\hline $\begin{array}{l}\% \\
\text { Columna }\end{array}$ & 15,3 & 9,1 & 13,0 & 7,6 & 20,0 & 31,4 \\
\hline
\end{tabular}

Por último, para contrastar las posibles diferencias en los tópicos de Gestión de Recursos Humanos, agrupados en las tres categorías anteriores, de acuerdo a las revistas consideradas se procedió mediante el test de la c2 de Pearson (Cuadro 7). Los resultados muestran la existencia de diferencias muy significativas en cuanto a la frecuencia con la que las revistas consideradas aluden a los distintos tipos de tópicos. Así, la Human Resource Management hace más hincapié en los nuevos tópicos, en una proporción similar a la que Capital Humano lo hace más sobre los tópicos tradicionales. Por lo que era lógico encontrar diferencias entre ambas revistas. 


\section{Cuadro 7. TEST $\mathbf{x}^{2}$ DE DIFERENCIAS EN LOS TÓPICOS DE GESTIÓN DE RECURSOS HUMANOS SEGÚN LAS REVISTAS CONSIDERADAS}

\begin{tabular}{|l|l|l|l|l|}
\hline & $\mathbf{n}$ & Valor de x2 & g.l. & p \\
\hline $\begin{array}{l}\text { Tópicos de } \\
\text { GRH }\end{array}$ & 1.193 & 53,882 & 2 & 0,000 \\
\hline
\end{tabular}

Se cumplen los supuestos de aplicación del test de c2 ya que ninguna casilla tienen una frecuencia esperada inferior a 5 y la frecuencia mínima esperada es 13.36.

\section{Conclusiones, Limitaciones Y Futtros Estudios}

Entre las principales conclusiones se puede mencionar que los años con un mayor número de artículos, y por lo tanto, se supone con un mayor interés por los temas relacionados con la Gestión de Recursos Humanos, son los de la mitad de la década de los noventa. No obstante, en el último año del período estudiado, se ha producido un repunte importante, tanto a nivel internacional como nacional.

En cuanto a los tópicos más tratados y su evolución, la formación es el más tratado a nivel nacional, ocupando también un lugar destacado a nivel internacional, ya que es el tercer tópico sobre el que más se ha insistido. Además, su evolución es relativamente constante en ambos ámbitos, incluso con un importante repunte en los últimos años. La calidad y la Gestión de Recursos Humanos, así como el liderazgo, igualmente, son tópicos ampliamente tratados. sobre todo, a nivel nacional. Sin embargo, su tendencia es decreciente, especialmente en el caso de la calidad, tanto a nivel nacional como internacional.

Las nuevas funciones asignadas a la gestión de recursos humanos y las nuevas competencias que deben poseer sus responsables es el tópico más tratado en la escena internacional, aunque también se ha insistido en él en España. Su evolución indica que se está produciendo un creciente interés en dichos ámbitos. La gestión estratégica de recursos humanos, también es un tema en el que se insiste de manera importante, sobre todo, a nivel internacional y en los últimos años de la década.

Todo lo anterior tiene su lógica, si pensamos que el típico departamento de recursos humanos, caracterizado por la asignación de tareas administrativas (cumplimentación de nóminas, contratos, etc.) debe asumir, además, otras funciones relacionadas con el apoyo que debe prestar a la dirección de la empresa en la formulación e implementación de su estrategia. En este nuevo escenario, la formación se constituye en el proceso de Gestión de Recursos Humanos más importante para llevar a cabo el cambio necesario en los trabajadores de la empresa (cultural, de conocimientos, competencias, etc.) a fin de afrontar, con ciertas garantías de éxito, 
las amenazas y oportunidades que provienen de un entorno cada vez más impredecible y sometido a cambios constantes, y por tanto, procurando aumentar las fortalezas y minimizar las debilidades de los componentes de la organización. En este sentido, como señala Valle (1995: pp. 102 y 103), los objetivos de la gestión estratégica de recursos humanos se denominan estratégicos por el importante papel que tienen en el proceso adaptativo de las empresas a su entorno.

Por último, se pone de manifiesto las diferencias apuntadas por Quintanilla (1991: pp. 22-24), en cuanto a que en España se sigue un modelo de Gestión de Recursos Humanos diferente al seguido a nivel internacional por los países más evolucionados en temas relacionados con esta disciplina. En concreto, en España todavía se sigue insistiendo mayoritariamente en los tópicos tradicionales, cuando en países donde la función de recursos humanos está más desarrollada se produce lo contrario, es decir, una mayor atención a los tópicos recientes. Las causas, pueden ser las apuntadas por Quintanilla, a saber: diferencias culturales que llevan a distintas formas de percepción y actuación. Aunque también habría que mencionar el retraso en la consideración de la Gestión de Recursos Humanos como una disciplina importante a tener en cuenta, tanto en el plano empresarial como académico. Así, el departamento de recursos humanos aparece como unidad diferenciada de otros departamentos (producción, marketing, etc.), aunque sin su mismo peso en fechas muy recientes, cuando en otros países ya se había colocado como un departamento de un peso similar al de los demás de la empresa. A nivel académico, la incorporación de la Gestión de Recursos Humanos, ni siquiera aparece como asignatura troncal en los planes de estudios relacionados con la empresa y en el mejor de los casos aparece como asignatura obligatoria de universidad o como optativa, caso de la asignatura que aspiramos cubrir.

Entre las limitaciones del presente estudio, entresacamos las que nos han parecido más importantes, en el sentido de que le restan validez científica a las conclusiones obtenidas. Entre ellas tenemos que hemos cogido tan sólo dos revistas representativas, una del ámbito nacional y otra del internacional, cuando igual podría haber sido también interesante tener en cuenta más revistas, como la de AEDIPE en España y Human Resource Planning a nivel internacional, pero con el fin de no extender en exceso este estudio, optamos por la alternativa seguida. Además, la revista de AEDIPE no aparece en la base de datos ISOC sobre revistas españolas. Otra importante limitación proviene de procedimiento de clasificación de los artículos, donde el carácter subjetivo de la misma, podría haber hecho incluir algunos artículos en una categoría que otro investigador no lo habría hecho. No obstante, la imposibilidad de utilizar las palabras clave de los artículos para su clasificación nos impidió proceder mediante este procedimiento, ya que para muchos de ellos no aparecían en las bases de datos consultadas.

Futuras lineas de investigación abiertas podrian profundizar en la solución de lás limitaciones antes apuntadas, incluso extender el estudio a un mayor número de años. 
BiBLIOGRAFIA

DOLAN, S.; SCHULER, R.S. y VALLE, R. (1999): La Géstión de los Recursos Humanos. Madrid, McGraw-Hill.

MATIAS, F. (2000): Las Empresas de Trabajo Temporal y la Gestion de Recursos Humanos de las Empresas Usuarias, Granada, Editorial L niversidad cle Granada.

MIRANDA, M. (1995): "Los Empleados del Futuro", Actualidad Económica. N. 16 de Enero, p. 8.

QUINTANILlA, I. (1991): Recursos Humanos y Marketing Interno. Madrid. Pirámide.

ROMAN, M. (2000): Proyecto Docente, Sevilla, Lniversidad de Sevilla.

ULRICH, D. (1997): "Judge Me More by My Future Than by My Past". Human Resource Management, Vol. 36, N. 1, pp. 5-8.

VALLE, R. (1995): La Gestión Estratégica de los Recursos Humanos, Delaware, Addison-Wesley Iberoamenticana. Wilmington. 